\title{
ALTITUD DEL LÍMITE SUPERIOR DE BOSQUE EN EL EJE NEOVOLCÁNICO MEXICANO, UN REFERENTE CLIMÁTICO DE LA ALTA MONTAÑA EN MÉXICO
}

\author{
Víctor Hugo Soto Molina \\ Universidad Veracruzana, Facultad de Ciencias Biológicas y Agropecuarias, Tuxpan, Ver, México \\ visoto@uv.mx \\ ORCID iD: http://orcid.org/0000-0003-1633-0752 \\ Juan Manuel Pech Canché \\ Universidad Veracruzana, Facultad de Ciencias Biológicas y Agropecuarias, Tuxpan, Ver, México \\ jmpech@uv.mx \\ ORCID iD: https://orcid.org/0000-0002-8455-593X \\ José Luís Alanís Méndez \\ Universidad Veracruzana, Facultad de Ciencias Biológicas y Agropecuarias, Tuxpan, Ver, México \\ lalanis@uv.mx \\ ORCID iD: https://orcid.org/0000-0002-2140-7139
}

Cómo citar este artículo/citation: Soto Molina, Víctor Hugo; Pech Canché, Juan Manuel y Alanís Méndez, José Luís (2021). Altitud del límite superior de bosque en el Eje Neovolcánico Mexicano, un referente climático de la alta montaña en México. Estudios Geográficos, 82 (290), e063. https://doi.org/10.3989/estgeogr.202075.075

RESUMEN: Se identificó el límite superior de bosque continuo y discontinuo en las montañas superiores a 4000 metros sobre el nivel del mar en México. El estudio se basó en el índice de vegetación de diferencia normalizada y mediante el análisis de componentes principales. Para el primer caso, se encontró que en cada vertiente el límite de bosque alcanza una altitud distinta; sin embargo, en promedio el bosque continuo culmina a 4019 y el discontinuo a 4072 msnm. Por su parte, el análisis estadístico permitió determinar que la precipitación, el tipo de suelo y la tasa de radiación solar a nivel de superficie son los principales factores que rigen su altitud. Finalmente se halló que el régimen de temperatura encontrado en esta frontera se aproxima a los valores estándares de la zona intertropical.

Palabras clave: Análisis de componentes principales; climatología de alta montaña; índice de vegetación de diferencia normalizada; límite superior de bosque; variabilidad climática.

\section{Altitude of the forest upper limit in the Mexican Neovolcanic Axis, a climate reference for high-moun- tain environment in Mexico}

ABSTRACT: The upper limit of continuous and discontinuous forest in mountains higher than 4000 meters above sea level in Mexico was identified. This study was based on the normalized difference vegetation index and by means of principal component analysis. For the first case, it was found that in each slope the forest limit reaches a different altitude; however, on average the continuous forest (timberline) ends at 4019 and the discontinuous forest (treeline) at 4072 masl. On the other hand, the statistical analysis made it possible to determine that precipitation, soil type and the rate of solar radiation at the surface level are the main factors that govern its altitude. Finally, it was found that the temperature regime found on this limit approximates the standard values of the intertropical zone.

Key words: Climatic variability; forest u pper limit; high-mountain climatology; normalized difference vegetation index; principal components analysis. 


\section{INTRODUCCIÓN}

El relieve mexicano es heterogéneo y muy accidentado. Más de dos terceras partes de su territorio está compuesto por regiones montañosas (Montero-García, 2004). Las principales provincias de montaña que posee son la Sierra Madre Oriental, Sierra Madre Occidental, Sierra Madre del Sur y el Eje Neovolcánico; este último cruza de este a oeste el país en su parte central y se caracteriza por poseer las cimas más altas de la nación. Datos del Instituto Nacional de Estadística, Geografía e Informática (INEGI, 2017) señalan que 31 montañas superan los 3000 metros sobre el nivel del mar (msnm), y entre ellas el Iztaccíhuatl (5220 msnm), Popocatépetl (5500 msnm) y Citlaltépetl (5610 msnm) superan la línea de nieves perpetuas, la cual para el caso de México está próxima a los 5000 msnm. No obstante a que estos tres volcanes poseen glaciares o zonas de nieve perenne, existen otras siete montañas por arriba de 4000 msnm que presentan distintos grados de acumulación nival durante el invierno, esto como producto del clima característico de alta montaña y del arribo de masas de aire polar, frecuentes durante los meses de noviembre a marzo de cada año (Jáuregui, 1975).

Las condiciones de temperatura en las altas montañas tropicales, con un valor medio anual $\leq 5^{\circ} \mathrm{C}$ rigen los constantes ciclos de helada muy recurrentes en altitudes superiores a los $4000 \mathrm{msnm}$. Este régimen térmico, desde la perspectiva mecánica, genera la crioclastia y favorece el flujo de escombros y la solifluxión en las laderas, que alteran o destruyen la vegetación juvenil de estas regiones de montaña (Hreško et al., 2009; French, 2018). Los ciclos de congelamiento superficial también afectan la fisiología vegetal; Wieser y Tausz (2007) señalan que la deficiencia de calor altera el metabolismo, el crecimiento y la regeneración de los árboles. Estas condiciones negativas pueden ser incluso mayores ante la presencia de nieve estacional con meses de permanencia. La combinación de estas circunstancias, entre otros factores, limitan o impiden la germinación y el crecimiento de la vegetación (Hatfield y Prueger, 2015), determinando así el límite altitudinal del bosque; el cual al menos para las montañas tropicales, particularmente las mexicanas, coincide generalmente con una temperatura media anual del aire de $5-6^{\circ} \mathrm{C}$ (Lauer y Klaus, 1975). Debido a que el régimen térmico del aire y el suelo están estrechamente correlacionados (Arya, 2001; Soto-Molina y Delgado-Granados, 2020), el suelo en el límite superior del bosque suele poseer un valor térmico anual de entre 6 y $7^{\circ} \mathrm{C}$ (Hoch y Körner, 2003) a $\sim 20 \mathrm{~cm}$ de profundidad; apenas un grado por encima del valor correspondiente al aire. Son estas condiciones térmicas, entre otros factores, las que regulan el límite superior del bosque en México.

Como se puede entender, el límite superior del boque es un ecotono donde la vegetación arbórea lucha por subsistir, adaptándose a un entorno de condiciones extremas (Turunen y Latola, 2005). Por arriba de su límite, la vegetación maderable es sustituida por especies de matorrales que se presentan de forma aislada hasta alcanzar la cota que impide el total crecimiento vegetal; en el volcán Citlaltépetl, por ejemplo, esta frontera se localiza a 4600 msnm (Lauer y Klaus, 1975; Soto-Molina y Delgado-Granados, 2020).

El cambio altitudinal del límite superior del bosque a lo largo del tiempo es un tema ampliamente documentado (Takahashi et al., 2012; Vincze et al., 2014; Schwörer et al., 2014), sobre todo en el marco actual de cambios evidentes en las condiciones normales del clima (Kaczka et al., 2015; Jandl et al., 2018; Chen et al., 2018), en el que la variación de los patrones de temperatura y de precipitación obligan a que este límite se ubique cada vez a mayor altitud (Santaella, 2013), tal como sucede con la isoterma $0^{\circ} \mathrm{C}$ o línea de nieves perpetuas. Una temperatura en aumento favorece, en parte, el crecimiento y extensión del bosque (Wang et al., 2006); y si el régimen de precipitación líquida supera al de la nieve, este avance altitudinal es aún más notorio. De este modo, el cambio altitudinal del límite de bosque funciona como un indicador más de cambio climático de mediano y largo plazo (Gaire et al., 2017).

De acuerdo con Ward (1994) y Gellhorn (2002), de manera paralela a la línea de las nieves, el límite superior del bosque desciende de manera regular a partir del ecuador, donde alcanza una altitud de 4500 msnm (Li, 1993), hasta llegar al nivel del mar en zonas circumpolares. No obstante, Holtmeier (2009) menciona que en el ecuador se forma un valle que está delimitado por las regiones de montañas subtropicales localizadas entre los $20^{\circ}$ y $30^{\circ}$ de latitud norte y sur, que es donde el límite de bosque alcanza su mayor altitud; a partir de estas crestas decrece hasta las zonas cercanas a los polos. Existen, sin embargo, algunas especies de Polylepis, que son beneficiadas a nivel de micro escala que pueden alcanzar una altitud de hasta $5400 \mathrm{msnm}$, como en el caso de Bolivia, marcando el límite superior de bosque más alto del planeta (Holtmeier, 2009). Por lo tanto, en términos generales, la latitud (Odland, 2015) es un factor que rige en gran medida el piso altitudinal del bosque. Al- 
gunas aproximaciones sobre el gradiente latitudinal del bosque señalan variaciones que van desde los 71 (Malyshev, 1993) hasta 184 m (Arno, 1984) por cada grado de latitud.

El límite superior de bosque continuo, según Holtmeier (2009), está determinado por la densidad de la vegetación y la altura de los individuos que lo componen, independientemente de las especies que lo integren. De acuerdo con la Organización de las Naciones Unidas para la Agricultura y la Alimentación (ONUAA, 2010), para identificar áreas de bosque desde la perspectiva de cobertura, la densidad del dosel debe ser superior al $10 \%$ y extenderse en una superficie mayor a 0,5 hectáreas. Por su parte, la altura de los individuos que lo integran debe ser de por lo menos $2 \mathrm{~m}$ (Hofgaard, 1997). Sin embargo, la altura mínima requerida puede ser un tanto arbitraria ya que depende de cada especie que integra las distintas áreas boscosas del planeta. A este respecto, Aas y Faarlund (1996) señalan una altura mínima de 2,5 m para la especie Betula tortuosa; Kullman (1987) indica 5 m para Pinus sylvestris, o hasta $8 \mathrm{~m}$ para Gynoxis y Hesperomeles (Salomons, 1986). Para el caso del bosque de gran altura en México, en el que según Gernandt y Pérez-de la Rosa (2014), existen familias como Pinacea y Cupressaceae, géneros como Abies, Juníperus y Pinus, su límite está compuesto principalmente por la especie Pinus hartwegii (Sánchez-González, 2008; Alfaro-Ramírez et al., 2017; Steinmann et al., 2019), aunque suelen encontrarse de forma intercalada individuos de Abies religiosa (Rzedowski, 2006); aquí el promedio mínimo de altura suele ser de 3-5 m.

Adicionalmente a las variables de temperatura y precipitación, existen otros factores ambientales que determinan la abundancia y distribución de las especies boscosas de alta montaña. La familia de Pinus obtiene mayor beneficio de un sustrato geológico compuesto por rocas ígneas; Rzedowski (2006) menciona que esto es debido a su mayor acidez, la cual se refleja en el tipo de suelos oscuros que la albergan (Aguilera et al., 1962). La mayoría de los suelos de alta montaña de latitudes medias y bajas son relativamente jóvenes, ya que evolucionaron a partir del retroceso glaciar del Holoceno (Holtmeier, 2009). De acuerdo con este autor, no existen estudios específicos de suelos en las montañas intertropicales, y la información habida es muy general. Por el contrario, en la región de los Alpes, donde se han realizado análisis a detalle, se han encontrado tipos de Andosoles, Regosoles, Cambisoles y Podzoles que están asociados a condiciones ambientales de micro escala. Con base en lo anterior, se puede señalar que no existe un suelo particular- mente definido en los límites boscosos.

Conforme aumenta la altitud el espesor de la atmósfera y su densidad es menor, e influye en la tasa de radiación solar a nivel de superficie. La radiación UV (especialmente la tipo B) determina en buena medida las condiciones del bosque superior debido al estrés fisiológico que le ocasiona, lo cual se traduce en una adaptación débil de las especies (Turunen y Latola, 2005). Bader et al., (2007) y Bader y Ruijten (2008) enfatizan lo anterior señalando que las bajas temperaturas, combinadas con un alto nivel de radiación solar resultan ser intolerables para las plántulas. Debido a que en el hemisferio boreal la mayor tasa de insolación se presenta en vertientes con dirección sur y oeste, la orientación del relieve y su pendiente tiene una influencia directa en la cantidad de radiación solar recibida en el suelo y por lo tanto, sobre la vegetación.

Otras variables, como la exposición a los vientos y la baja presión atmosférica son también elementos que determinan, aunque en menor medida que los anteriores, la presencia y la altitud del límite de bosque de montaña. Las laderas expuestas al viento experimentan mayor déficit de temperatura del suelo y del aire que los sitios mejor protegidos (Dahms, 1992); esto es principalmente ocasionado por la sensación térmica, la cual se relaciona con la velocidad del viento. Mecánicamente, la presión de viento y la abrasión también afectan el crecimiento vegetal (Holtmeier, 2009). La ausencia de materia orgánica en el suelo, como fuente de carbono, limita también su cota altitudinal (Cairns, 1998).

Debido a las características montañosas del relieve mexicano, y por la importancia del límite superior de bosque como indicador de cambio climático de mediano y largo plazo, este trabajo está enfocado en determinar el límite de bosque en las montañas mexicanas superiores a $4000 \mathrm{msnm}$. Este trabajo fortalece el conocimiento de las condiciones actuales de altitud del bosque de la alta montaña mexicana, el cual puede ser útil como referencia en temas de clima y vegetación de gran altura, no sólo en el país, sino también en otras regiones de montañas tropicales.

\section{ÁREA DE ESTUDIO}

El Eje Neovolcánico es una franja de montañas y mesetas ubicada alrededor de los $19,5^{\circ}$ de latitud norte. Su longitud es de $\sim 900 \mathrm{~km}$, posee un ancho promedio de $150 \mathrm{~km}$ y cruza el país de este a oeste abarcando distintos estados, entre éstos la capital de la nación. 
En su interior se localizan las cimas más altas del país que están separadas por altiplanicies que poseen una altitud media de 2000 msnm. Está integrado por distintos volcanes monogenéticos y estratovolcanes. Algunos están extintos, otros en etapa de reposo y otros más en fase de actividad eruptiva, como el volcán de Fuego de Colima y el Popocatépetl. De acuerdo con Demant (1978), la edad de esta franja volcánica está datada dentro del periodo Plio-Cuaternario; aunque para Ferrari et al., (2012) existen indicios de evolución a partir de principios del Mioceno, hace $\sim 19$ millones de años. El lecho rocoso en sus partes más altas está compuesto en su mayoría por dacitas, andesitas y riolitas, producto de los distintos episodios eruptivos a lo largo de su historia.

El clima es variado en función de la continentalidad y de la altura del relieve. Con base en García (2004), en las partes próximas a las costas, donde la humedad oceánica influye de forma directa, varía desde semicálido con un alto índice pluvial en altitudes inferiores a $800 \mathrm{msnm}$, con una temperatura media anual que oscila entre 18 y $22^{\circ} \mathrm{C}$. Por encima de esta altitud inicia la presencia de bosque mesófilo con un clima templado y húmedo que culmina hasta los 2000 msnm. La temperatura anual de esta zona es inferior a $18^{\circ} \mathrm{C}$ y hay registros pluviales, aunque de manera aislada, que llegan a alcanzar hasta $4500 \mathrm{~mm}$ al año; sin embargo, el resto de este rango altitudinal posee una media de $2500 \mathrm{~mm}$. Más arriba de esta cota la temperatura disminuye y también el régimen de precipitación. Aquí se inicia generalmente el bosque de pino-encino que alcanza cotas cercanas a $3400 \mathrm{msnm}$ y el de pino limita a $\sim 100$ en función de distintos rasgos topo-climáticos. El clima es de tipo semifrío con una temperatura media anual de $8^{\circ} \mathrm{C}$. La precipitación alcanza valores comúnmente menores a $1200 \mathrm{~mm}$ al año. Los climas fríos de tundra o de hielos perpetuos se inician por arriba de este límite; el primero de estos posee un régimen térmico que oscila entre -2 y $5^{\circ} \mathrm{C}$; su límite se ubica aproximadamente a $4000 \mathrm{msnm}$. Por su parte, el clima más frío de todos se localiza a 5100 $\mathrm{msnm}$ con un valor térmico anual inferior a $-2^{\circ} \mathrm{C}$. Por otro lado, las regiones de altiplanicie, ubicadas en el centro del Eje Neovolcánico y alejadas de la influencia

FIGURA1

ZONA DE ESTUDIO

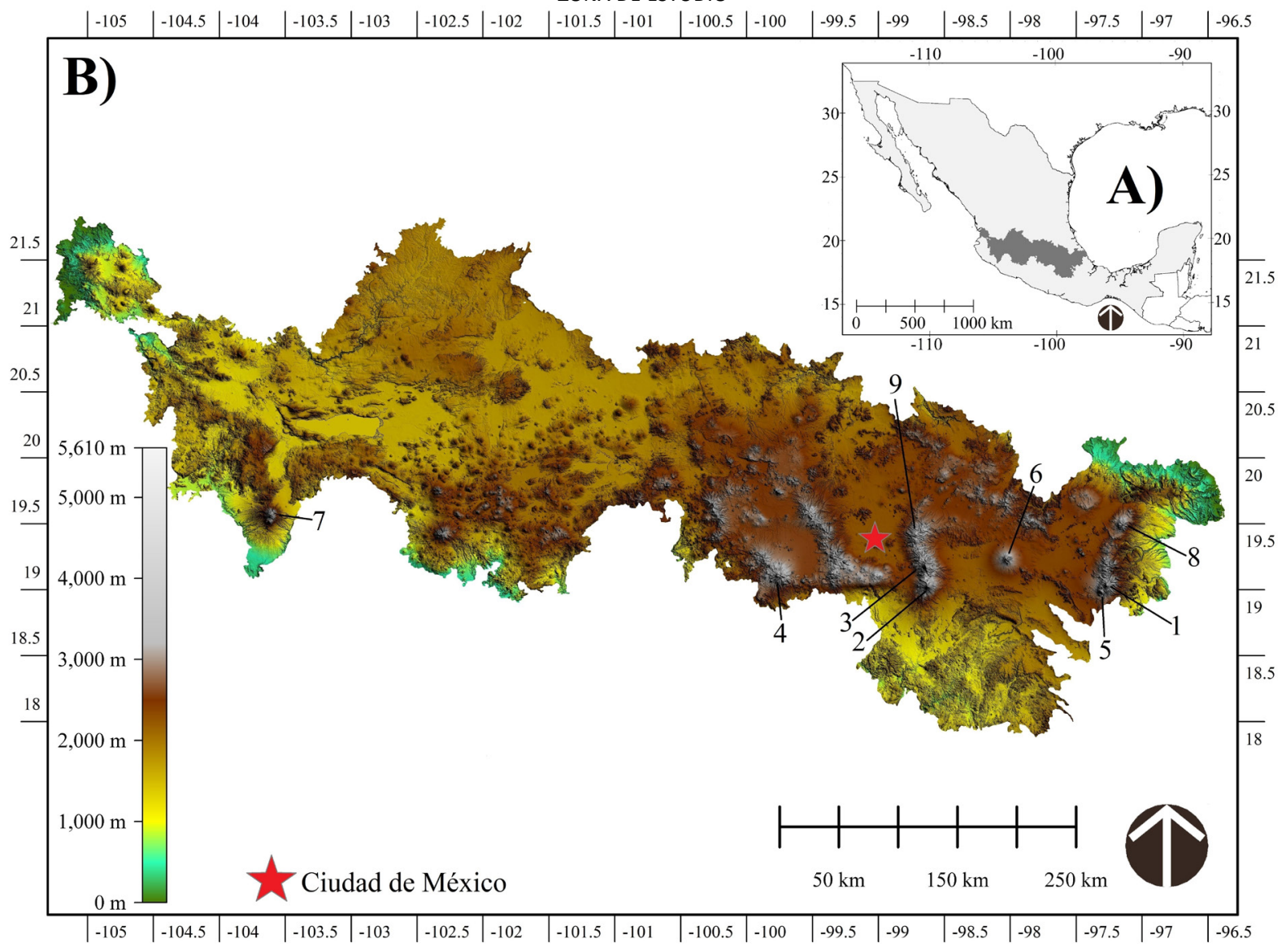


oceánica son en su mayoría de clima templado con una temperatura media anual de 18 a $22^{\circ} \mathrm{C}$ pero con menor cantidad de precipitación en comparación con las zonas de montaña. En las mesetas centrales existen también amplias áreas que poseen climas secos de estepa; en parte esto es consecuencia de los vientos catabáticos que dan origen al efecto Foehn en regiones adyacentes a zonas de montaña.

La figura y la tabla 1 señalan la distribución de las montañas que superan los $4000 \mathrm{msnm}$ con sus altitudes correspondientes según información del INEGI TABLA 1

MONTAÑAS SUPERIORES A 4000 MSNM

\begin{tabular}{|l|l|l|l|l|}
\hline $\begin{array}{c}\text { Lugar en } \\
\text { altitud }\end{array}$ & \multicolumn{1}{|c|}{ Montaña } & \multicolumn{1}{|c|}{ Altitud } & \multicolumn{1}{c|}{$\begin{array}{c}\text { Latitud } \\
\text { Norte }\end{array}$} & $\begin{array}{c}\text { Longitud } \\
\text { Oeste }\end{array}$ \\
\hline 1 & Citlaltépetl & 5610 & $19^{\circ} 02^{\prime} 00^{\prime \prime}$ & $97^{\circ} 16^{\prime} 12^{\prime \prime}$ \\
\hline 2 & Popocatépetl & 5500 & $19^{\circ} 01^{\prime} 21^{\prime \prime}$ & $98^{\circ} 37^{\prime} 39^{\prime \prime}$ \\
\hline 3 & Iztaccíhuatl & 5220 & $19^{\circ} 11^{\prime} 00^{\prime \prime}$ & $98^{\circ} 38^{\prime} 36^{\prime \prime}$ \\
\hline 4 & Xinantécatl & 4680 & $19^{\circ} 06^{\prime} 19^{\prime \prime}$ & $99^{\circ} 46^{\prime} 09^{\prime \prime}$ \\
\hline 5 & Sierra Negra & 4580 & $18^{\circ} 59^{\prime} 05^{\prime \prime}$ & $97^{\circ} 19^{\prime} 00^{\prime \prime}$ \\
\hline 6 & Matlalcuéyetl & 4420 & $19^{\circ} 14^{\prime} 00^{\prime \prime}$ & $98^{\circ} 02^{\prime} 00^{\prime \prime}$ \\
\hline 7 & Nevado de Colima & 4260 & $19^{\circ} 33^{\prime} 45^{\prime \prime}$ & $103^{\circ} 36^{\prime} 31^{\prime \prime}$ \\
\hline 8 & Naucampatépetl & 4200 & $19^{\circ} 29^{\prime} 04^{\prime \prime}$ & $97^{\circ} 09^{\prime} 37^{\prime \prime}$ \\
\hline 9 & Tláloc & 4120 & $19^{\circ} 24^{\prime} 44^{\prime \prime}$ & $98^{\circ} 42^{\prime} 45^{\prime \prime}$ \\
\hline 10 & Tacaná* & 4080 & $15^{\circ} 07^{\prime} 56^{\prime \prime}$ & $92^{\circ} 06^{\prime} 30^{\prime \prime}$ \\
\hline
\end{tabular}

*No considerada en el estudio debido a su latitud.

Fuente: elaboración propia.

(2017).

Es importante señalar que el volcán Tacaná (número 10) no ha sido tomado en cuenta en este estudio debido a que, no obstante su altitud de $4080 \mathrm{msnm}$, su ubicación a $15^{\circ}$ de latitud ocasiona que su cima se encuentre cubierta por bosque de pinos, enfatizando con esto la teoría del gradiente latitudinal de la línea de bosque previamente señalado.

\section{FUENTES Y METODOLOGÍA}

Los sensores remotos son una herramienta ampliamente utilizada en la actualidad para el estudio de la superficie terrestre y su vegetación, entre otros elementos ambientales. Para observar las condiciones de la vegetación, y de manera particular delimitar la línea de bosque, es muy recurrido el uso de imágenes satelitales de distintos sensores como AVHRR (Kogan et al., 2003), Modis (Danzeglocke, 2005), Aster (Sitko y Troll, 2008), y más comúnmente los de la serie Landsat (Nelson et al., 2009; Bharti et al., 2011; Braunisch et al., 2016) entre otros. Sin embargo, debido a su mayor resolución espacial (10 m por pixel en las bandas 2 a 8), las imágenes Sentinel-2 (A y B) son de momento las que ofrecen mayor precisión en los resultados dentro de las imágenes de acceso gratuito. Una más de las ventajas ofrecidas por la serie de imágenes Sentinel-2, radica en que su nivel de procesamiento (a partir del nivel $1 C$ ) posee correcciones radiométricas y geométricas que incluyen orto-rectificación y registro espacial en un sistema de referencia global con precisión de subpíxel (ESA, 2015). No obstante lo anterior, para esta investigación se realizó un nuevo procesamiento de corrección radiométrica a la base de la atmósfera mediante la herramienta Sen2cor del programa SNAP, de la Agencia Espacial Europea (ESA), para lograr una mayor precisión mediante la eliminación del efecto de aberración causado por los aerosoles atmosféricos ( $\mathrm{Li}$ et al., 2018).

Por otra parte, el empleo del índice de vegetación de diferencia normalizada (NDVI en inglés) es un parámetro muy utilizado para identificar la calidad de la vegetación y su densidad (Zaitunah et al., 2018); adicionalmente permite distinguir la cobertura vegetal del suelo desnudo, ya que una de sus ventajas radica en la facilidad de cuantificar las diferencias representadas mediante cada pixel (Singh et al., 2012). Su rango va de $-1 \mathrm{a}+1$, siendo los valores cercanos al -1 los correspondientes a los cuerpos de agua, y los valores próximos a 0 representan el suelo desnudo. Los valores positivos indican las diferentes cualidades de la vegetación; un valor cercano a +1 indica la presencia de una vegetación vigorosa y densa. De este modo, en una imagen de alta resolución espacial, como las Sentinel-2 en este caso, cada pixel de 10×10 $\mathrm{m}$ representaría el dosel forestal con algunos metros de cobertura. Dadas estas propiedades de resolución, en este trabajo se elaboró un mosaico de imágenes Sentinel-2A correspondiente al mes de abril del 2019 con el que se cubrieran las montañas mayores a 4000 msnm dentro del Eje Neovolcánico. Las imágenes fueron obtenidas de la plataforma Earth Explorer de la USGS (2019) de Estados Unidos. Se procuró obtener la serie de imágenes más recientes dentro del período de estiaje con la finalidad de que estuvieran libres de nubosidad, así como también para evitar la sobreestimación ocasionada por el exceso de humedad durante la temporada de lluvias. De este modo, la tabla 2 muestra las imágenes seleccionadas para este trabajo.

Para el cálculo del NDVI, las imágenes fueron procesadas dentro del programa Global Mapper v. 20 a través de la combinación de las bandas 4 y 8 (Delegido et al., 2016; Arekhi et al., 2019) mediante el algoritmo NIR - Red
NIR + Red pectral del rojo (Red) y la banda 8 al infrarrojo cercano $(N I R)$; ambas con resolución espacial de $10 \mathrm{~m}$ por pi- 
TABLA 2

CONJUNTO DE IMÁGENES SENTINEL-2A EMPLEADAS PARA EL CÁLCULO DEL NDVI

\begin{tabular}{|l|l|}
\hline \multicolumn{1}{|c|}{ Imagen } & \multicolumn{1}{c|}{ Fecha de la toma } \\
\hline T13QFB_20190423T171901 & 23 de abril 2019 \\
\hline T14QMG_20190412T165849 & 12 de abril 2019 \\
\hline T14QNG_20190412T165849 & 12 de abril 2019 \\
\hline T14QPG_20190330T164859 & 30 de marzo 2019 \\
\hline
\end{tabular}

Fuente: elaboración propia.

xel. Una vez generada la capa del NDVI, se consideró que la cobertura de pixeles que fuera superior al $10 \%$ del área, según el criterio de la ONUAA (2010), corresponderían a zonas de bosque continuo (timberline en inglés). De esta manera, la posición más alta con respecto al relieve, que tuviera una cobertura de pixeles superiores al $10 \%$ del área correspondiente señaló el límite altitudinal del bosque continuo. La cobertura inferior al $10 \%$, por su parte, representó los parches discontinuos o individuos aislados del bosque (treeline en inglés).

De las capas ráster resultantes se eliminaron los valores que correspondieron al lecho rocoso y suelo desnudo (NDVI $\leq 0$ ) y de vegetación de baja densidad $(0<\mathrm{NDVI}<0,3)$, pertenecientes a la vegetación no arbórea, para que los pixeles correspondientes a la vegetación forestal, por arriba del valor de 0,3 (Reddy y Reddy, 2013; Malini y Somashekar, 2013; Zaitunah et al., 2018) quedaran aislados. Posteriormente esta capa se sobrepuso a un modelo digital de elevaciones (MDE) de $15 \mathrm{~m}$ de resolución que fue obtenido de la página oficial del INEGI (2019), para identificar la altitud a la que se ubicaran los pixeles de vegetación; determinando así la altitud máxima de la línea de bosque continuo o timberline para cada una de las vertientes norte, sur, este y oeste de todas las montañas analizadas. De igual manera, se determinó la altitud máxima alcanzada por individuos aislados, lo que supuso el límite superior de bosque discontinuo (treeline).

Para identificar el grado de incidencia de los factores ambientales que condicionan la altitud de la línea superior de bosque, se elaboró un análisis de componentes principales por medio del programa SPSS v. 23, mediante el cual se correlacionaron las variables de 1. Orientación, 2. Temperatura, 3. Precipitación, 4. Lecho rocoso, 5. Tipo de suelo, 6. Evaporación y 7. Radiación. La utilidad del análisis de componentes principales se basa en la capacidad de identificar relaciones generando combinaciones lineales de variables que muestran tendencias comunes de variación, y puede contribuir sustancialmente al reconocimiento de patrones en el comportamiento de los datos analizados (Peres-Neto et al., 2005). Para los casos de temperatura, precipitación y evaporación, se consideraron los valores medios anuales, obtenidos de la plataforma digital de la Comisión Nacional para el Conocimiento y Uso de la Biodiversidad (CONABIO, 2019), al igual que las capas de roca y de suelo. Los valores de temperatura, precipitación y evaporación anuales fueron determinados a la altitud de 4000 msnm. Por su parte, el cálculo de la radiación solar incidente en superficie, también a escala anual, se obtuvo mediante la herramienta solar radiation del programa ArcGis v. 10.4 y el MDE. La radiación solar incidente se calculó a 4000 msnm en pendientes de $15^{\circ}$ para los acimuts $0^{\circ}, 90^{\circ}$, $180^{\circ}$ y $270^{\circ}$, que corresponde a los cuatro puntos cardinales.

El factor latitud no fue considerado en este trabajo debido a que todas las montañas analizadas oscilan al-

TABLA 3

VARIABLES Y RANGOS UTILIZADOS EN EL ANÁLISIS DE COMPONENTES PRINCIPALES

\begin{tabular}{|c|c|c|c|c|c|c|}
\hline Tipo principal de roca & $\begin{array}{l}\text { Tipo principal de } \\
\text { suelo } \\
\end{array}$ & $\begin{array}{c}\text { Precipitación anual } \\
(\mathrm{mm})\end{array}$ & $\begin{array}{c}\text { Temperatura anual } \\
\left({ }^{\circ} \mathrm{C}\right)\end{array}$ & $\begin{array}{c}\text { Radiación solar (W/ } \\
\mathrm{m} 2 / \mathrm{hr})\end{array}$ & Evaporación (mm) & $\begin{array}{c}\text { Orientación } \\
\text { (Grados) }\end{array}$ \\
\hline Toba ácida & Regosol éutrico & 700 & 5,0 & 238 & 550 & $315-45$ \\
\hline $\begin{array}{l}\text { Brecha volcánica } \\
\text { intermedia }\end{array}$ & Andosol ócrico & 900 & 5,3 & 267 & 600 & $45-135$ \\
\hline Brecha sedimentaria & Regosol dístrico & 1000 & 5,5 & 258 & 650 & $135-225$ \\
\hline Andesita & Litosol & 1100 & 5,8 & 252 & 700 & $225-315$ \\
\hline & Cambisol éutrico & 1350 & 6,3 & & 750 & \\
\hline & Andosol húmico & 1225 & 7,0 & & & \\
\hline & & 1650 & 8,0 & & & \\
\hline & & & 9,0 & & & \\
\hline & & & 10,0 & & & \\
\hline & & & 11,0 & & & \\
\hline
\end{tabular}

Fuente: elaboración propia. 
rededor de los $19^{\circ}$ y se encuentran relativamente muy próximas entre sí, por lo que, si bien está documentada su influencia en el límite altitudinal de la vegetación, en este caso su variabilidad entre una montaña y otra no representa diferencias significativas. Por lo tanto, considerando los elementos mencionados, se pudieron identificar los principales factores que pudieran determinar la posición del límite de bosque en cada una de las cuatro vertientes de las montañas del Eje Neovolcánico. La tabla 3 muestra los distintos parámetros que componen cada una de las variables analizadas.

\section{RESULTADOS}

Mediante el procedimiento para la obtención del NDVI, la figura 2 muestra un acercamiento a la imagen satelital en color verdadero (RGB) de la parte note del volcán Xinantécatl (figura $2 \mathrm{~A}$ ), así como los valores correspondientes del NDVI (figura 2 B). La comparación NDVI-RGB ha sido empleada en la mayoría de los estudios de cobertura forestal para fines de corrobo-

\section{FIGURA 2}

IMAgen SENTINEL-2A EN RGB (A) Y DEL NDVI (B) EN LA ZONA NORTE DEL VOLCÁN XINANTÉCATL. LOS VALORES MENORES A 0 PERTENECEN A CUERPOS DE AGUA EN EL INTERIOR DE CRÁTER
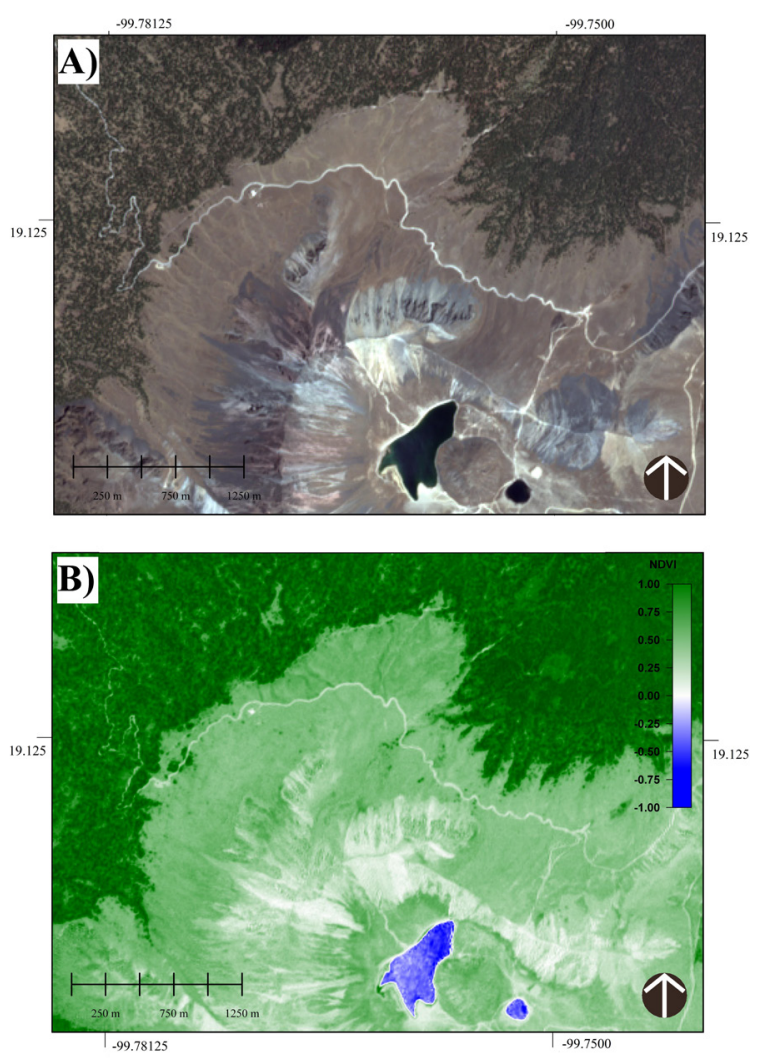

ración (Sader y Winne, 1992).

En la figura 2 se aprecia con nitidez cómo los pixeles de $10 \times 10 \mathrm{~m}$ del NDVI corresponden respectivamente a cada uno de los de la imagen RGB, en la que se representa la vegetación pinácea. Por lo tanto, debido al contraste entre pixeles de vegetación densa y del suelo desnudo, puede observarse con claridad el límite superior del bosque.

Tras la extracción de pixeles con valor superior a 0,3 del NDVI, la figura 3 señala la capa de cobertura de bosque continuo, la cual se ha sobrepuesto a un MDE

FIGURA 3

CAPA DE COBERTURA DE BOSQUE CONTINUO SOBRE EL MDE. LAS ISOHIPSAS, COMO REFERENCIA, ESTÁN EN INTERVALOS DE 10 METROS. LOS VALORES DE NDVI POR DEBAJO DE - 0.25 CORRESPONDEN A LOS LAGOS DEL SOL Y LA LUNA, EN EL CRÁTER DEL VOLCÁN

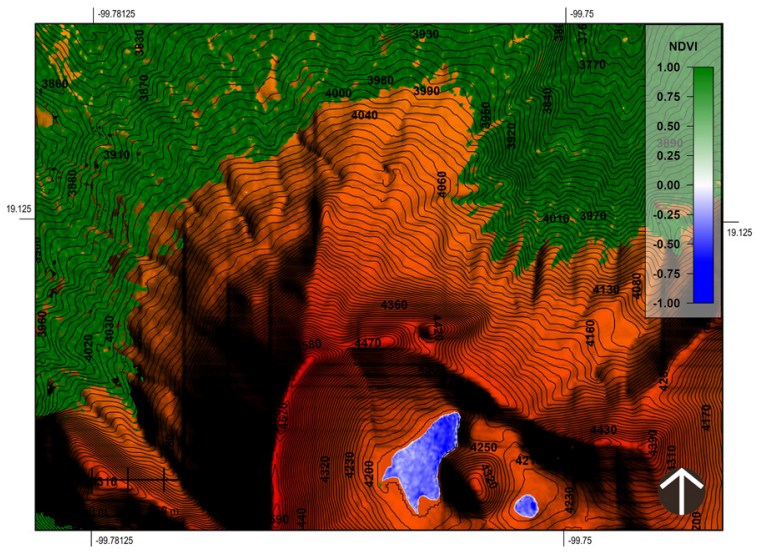

en que se observan los valores de altitud en los que se posiciona el límite superior.

En la figura 3 puede apreciarse con claridad la cota altitudinal que alcanza el bosque continuo y los individuos aislados conforme al MDE. Este ejemplo corresponde a la vertiente norte del volcán Xinantécatl. Pueden observarse también porciones de vegetación no arbórea (NDVI $\leq 0,3$ ), probablemente de tipo pastizal, o que corresponden a suelo desnudo (NDVI 0) entre el bosque de pinos. Una vez elaborados los procedimientos anteriores para las nueve montañas del Eje Neovolcánico, la tabla 4 muestra los valores de altitud por vertiente, así como los valores promedios para la cubierta continua y discontinua de bosque.

Con base en el resumen de la tabla 4, se tiene entonces que los límites superiores de bosque son distintos para cada ladera; de igual manera, su altitud es diferente en cada una de las montañas. Puede obser- 
TABLA 4

ALTITUD DEL LÍMITE SUPERIOR DE BOSQUE CONTINUO Y DISCONTINUO POR VERTIENTE

\begin{tabular}{|c|c|c|c|c|c|c|}
\hline Lugar en altitud & Montaña & Vertiente & Discontinuo & Promedio & Continuo & Promedio \\
\hline \multirow{4}{*}{1} & \multirow{4}{*}{ Citlaltépetl } & norte & 4100 & \multirow{4}{*}{4117,5} & 4030 & \multirow{4}{*}{4047,5} \\
\hline & & sur & 4200 & & 4120 & \\
\hline & & este & 4130 & & 4060 & \\
\hline & & oeste & 4040 & & 3980 & \\
\hline \multirow{4}{*}{2} & \multirow{4}{*}{ Popocatépetl } & norte & 4030 & \multirow{4}{*}{3985} & 4010 & \multirow{4}{*}{3935} \\
\hline & & sur & 4020 & & 3980 & \\
\hline & & este & 4010 & & 3910 & \\
\hline & & oeste & 3880 & & 3840 & \\
\hline \multirow{4}{*}{3} & \multirow{4}{*}{ Iztaccíhuat| } & norte & 4190 & \multirow{4}{*}{4170} & 4150 & \multirow{4}{*}{4122,5} \\
\hline & & sur & 4150 & & 4130 & \\
\hline & & este & 4200 & & 4170 & \\
\hline & & oeste & 4140 & & 4040 & \\
\hline \multirow{4}{*}{4} & \multirow{4}{*}{ Xinantécatl } & norte & 4100 & \multirow{4}{*}{4112,5} & 4090 & \multirow{4}{*}{4090} \\
\hline & & sur & 4130 & & 4070 & \\
\hline & & este & 4110 & & 4110 & \\
\hline & & oeste & 4110 & & 4090 & \\
\hline \multirow{4}{*}{5} & \multirow{4}{*}{ Sierra Negra } & norte & 4020 & \multirow{4}{*}{4102,5} & 3950 & \multirow{4}{*}{4035} \\
\hline & & sur & 4170 & & 4080 & \\
\hline & & este & 4240 & & 4210 & \\
\hline & & oeste & 3980 & & 3900 & \\
\hline \multirow{4}{*}{6} & \multirow{4}{*}{ Matlalcuéyetl } & norte & 4040 & \multirow{4}{*}{4055} & 4020 & \multirow{4}{*}{4002,5} \\
\hline & & sur & 4050 & & 3990 & \\
\hline & & este & 4060 & & 4050 & \\
\hline & & oeste & 4070 & & 3950 & \\
\hline \multirow{4}{*}{7} & & norte & 3900 & & 3860 & \\
\hline & De & sur & 3990 & זרח & 3900 & ז0רט \\
\hline & INevado de Colıma & este & 4050 & 3982,5 & 3970 & 3925 \\
\hline & & oeste & 3990 & & 3970 & \\
\hline & & norte & 4070 & & 4000 & \\
\hline $\mathrm{L}_{0}$ & & sur & 4050 & $1000 \Omega 5$ & 4010 & \\
\hline 8 & Naucampatepetl & este & 4060 & 4062,5 & 3900 & 3987,5 \\
\hline & & oeste & 4070 & & 4040 & \\
\hline & & norte & 4040 & & 4030 & \\
\hline$\rho_{0}$ & $T H t_{-1}$ & sur & 4100 & $D_{1000}$ & 4030 & 5025 \\
\hline 9 & Haloc & este & 4080 & 14060 & 4040 & 4025 \\
\hline & & oeste & 4020 & & 4000 & \\
\hline & & norte & 4054.5 & & 4015.5 & \\
\hline 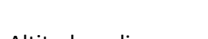 & & sur & 4095.5 & דר & 4034.4 & \\
\hline Altitud media por ve & ente & este & 4104.5 & $40 / 2$ & 4046.7 & 4018,9 \\
\hline & & oeste & 4033.3 & & 3978.9 & \\
\hline
\end{tabular}

Fuente: elaboración propia.

varse que el límite más bajo, en promedio, corresponde a las laderas oeste, con 4033,3 y 3978,9 msnm para el bosque discontinuo y continuo, respectivamente. Por su parte, las vertientes este son las que poseen un límite superior más alto, con 4104,5 msnm para el bosque discontinuo y 4046,7 para el bosque conti- nuo. De manera individual, la montaña con mayor altitud de cobertura boscosa es el Iztaccíhuatl, con 4170 msnm para la cubierta aislada y 4122,5 para el bosque continuo. Contrariamente, en el Nevado de Colima se encontró una altitud de 3982,5 y 3925 msnm para el bosque aislado y el continuo, respectivamente. 
Por su parte, el análisis de componentes principales permitió identificar los factores que condicionan la altitud del límite del bosque. De acuerdo con la prueba de significancia de la tabla 5 , se observa que existe un grado de explicación no muy alto $(0,532)$ pero con alto

TABLA 5

PRUEBa de SIGNIFICANCIA SEGÚN TEST DE ESFERICIDAD DE BARTLETT

\begin{tabular}{|l|l|l|}
\hline \multicolumn{3}{|c|}{ Prueba de KMO y Bartlett } \\
\hline Medida Kaiser-Meyer-Olkin de adecuación de muestreo & 0,532 \\
\hline \multirow{3}{*}{ Prueba de esfericidad de Bartlett } & Aprox. Chi-cuadrado & 69,262 \\
\cline { 2 - 3 } & gl & 21 \\
\cline { 2 - 3 } & Sig. & 0,000 \\
\hline
\end{tabular}

Fuente: elaboración propia.

nivel de significancia según la prueba de esfericidad de Bartlett, considerando un valor alfa de 0,05.

Mediante los resultados de la tabla 6 se determinó

FIGURA 4

IDENTIFICACIÓN DEL NÚMERO DE COMPONENTES QUE CONDICIONAN LA ALTITUD DEL LÍMITE SUPERIOR DEL BOSQUE

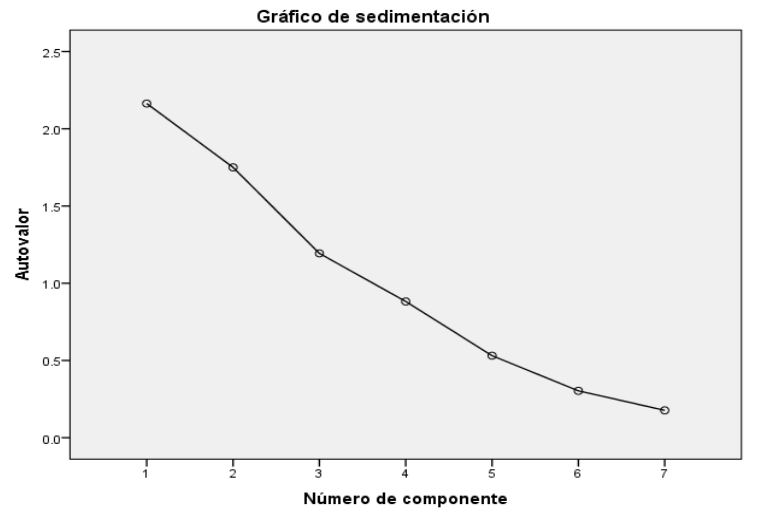

que son tres componentes principales los que condicionan la altitud del límite del bosque, que en conjunto alcanzan a explicar el fenómeno en un $73 \%$.

Lo anterior es mostrado en el gráfico de sedimentación (figura 4), donde se observa que al menos tres componentes superan el umbral de auto valor 1 .

Analizando cada uno de los componentes, según las variables con mayor nivel de incidencia (tabla 7), se puede determinar, de mayor a menor grado de importancia, que el factor principal, componente 1 , está compuesto por la variable de precipitación, seguida por la del tipo de

TABLA 7

NIVEL DE CONDICIONAMIENTO, SEGÚN LOS FACTORES IDENTIFICADOS

\begin{tabular}{|l|l|l|l|}
\hline Matriz de componente rotado \\
\hline & \multicolumn{4}{l}{ Componente } \\
\cline { 2 - 4 } & 1 & 2 & 3 \\
\hline PRECIPITACIÓN & 0,901 & & \\
\hline SUELO & 0,792 & & 0,322 \\
\hline EVAPORACIÓN & 0,594 & 0,240 & $-0,140$ \\
\hline RADIACIÓN & & 0,942 & 0,103 \\
\hline ORIENTACIÓN & & $-0,927$ & \\
\hline ROCA & 0,285 & & 0,813 \\
\hline TEMPERATURA & $-0,394$ & 0,191 & 0,646 \\
\hline $\begin{array}{l}\text { Método de extracción: análisis de componentes principales. } \\
\text { Método de rotación: Varimax con normalización Kaiser. }\end{array}$ \\
\hline a. La rotación ha convergido en 5 iteraciones. \\
\hline
\end{tabular}

Fuente: elaboración propia.

suelo; siendo éstos los elementos más determinantes en la altitud del bosque, seguido por el grado de radiación solar incidente como factor determinante 2 . En menor medida influye el tipo de roca y la temperatura.

De manera gráfica, la figura 5 muestra la posición de cada factor que interviene en el ejercicio, según su grado de importancia y su nivel de intensidad.

TABLA 6

PORCENTAJE DE EXPLICACIÓN POR LOS TRES COMPONENTES PRINCIPALES

\begin{tabular}{|c|c|c|c|c|c|c|c|c|c|}
\hline \multicolumn{10}{|c|}{ Varianza total explicada } \\
\hline \multirow{2}{*}{ Componente } & \multicolumn{3}{|c|}{ Autovalores iniciales } & \multicolumn{3}{|c|}{ Sumas de extracción de cargas al cuadrado } & \multicolumn{3}{|c|}{ Sumas de rotación de cargas al cuadrado } \\
\hline & Total & $\%$ de varianza & $\%$ acumulado & Total & $\%$ de varianza & $\%$ acumulado & Total & $\%$ de varianza & $\%$ acumulado \\
\hline 1 & 2,164 & 30,907 & 30,907 & 2,164 & 30,907 & 30,907 & 2,041 & 29,153 & 29,153 \\
\hline 2 & 1,750 & 25,001 & 55,908 & 1,750 & 25,001 & 55,908 & 1,850 & 26,429 & 55,582 \\
\hline 3 & 1,193 & 17,045 & 72,953 & 1,193 & 17,045 & 72,953 & 1,216 & 17,371 & 72,953 \\
\hline 4 & 0,882 & 12,601 & 85,553 & & & & & & \\
\hline 5 & 0,531 & 7,585 & 93,139 & & & & & & \\
\hline 6 & 0,303 & 4,335 & 97,474 & & & & & & \\
\hline 7 & 0,177 & 2,526 & 100,000 & & & & & & \\
\hline
\end{tabular}

Fuente: elaboración propia. 
FIGURA 5

GRÁFICA DE DISPERSIÓN TRIDIMENSIONAL POR COMPONENTE

Gráfico de componente en espacio rotado

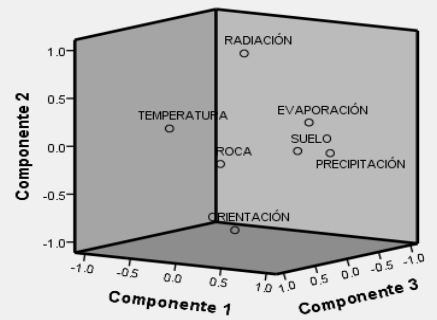

\section{DISCUSIÓN}

La altitud del límite bosque en México depende de la combinación de distintos factores que influyen de diferente manera en cada montaña del país; esto, aunado a la información no detallada de las variables medioambientales que intervienen en su delimitación, limitan la identificación precisa de los factores condicionantes. Con base en lo anterior, este trabajo resalta la necesidad de realizar análisis a detalle in situ para conocer las propiedades particulares de las variables aquí estudiadas para cada una de las montañas mexicanas superiores a $4000 \mathrm{msnm}$. Es probable que la respuesta del modelo de componentes principales (KMO $=0,532)$ se deba a la carencia de información más detallada de las variables analizadas. No obstante lo anterior, el alto nivel de significancia, según la prueba de Bartlett, con este trabajo se encontró que el límite altitudinal del límite de bosque en México depende principalmente de la precipitación, seguido del tipo del suelo y del grado de radiación solar a nivel de superficie.

En principio el factor precipitación, como elemento vital, queda de manifiesto en este trabajo al ser identificado como el más influyente en la determinación del límite altitudinal del bosque. Las propiedades físicoquímicas del suelo es el segundo elemento condicionante como lo señalan Aguilera et al., (1962). El tercer condicionante, la radiación solar, es también constatado por Turunen y Latola (2005), Bader et al., (2007) y Bader y Ruijten (2008). Las características del lecho rocoso y la temperatura del aire son factores que influyen en el alcance vertical de la vegetación arbórea pero en menor medida que los anteriores. Debido a la estrecha relación evolutiva entre la roca y el suelo, se entiende entonces que el lecho rocoso adquiere importancia como elemento determinante del límite superior arbóreo, sólo a través del tiempo y mediante su meteorización y transformación en suelos más fértiles, con la combinación de materia orgánica de origen vegetal, así como por los depósitos de ceniza volcánica. Por su parte, la variable de temperatura, si bien queda identificada también dentro del tercer componente y con el menor grado de incidencia, permite dejar en claro que la radiación solar a nivel de superficie, desde la perspectiva de la micrometeorología, es el componente atmosférico que determina la temperatura del suelo y como consecuencia del aire, mediante los flujos de calor sensible; esto dentro del balance de energía superficial, tal como lo manifiesta Arya (2001). Por lo tanto, la temperatura y el tipo de roca están estrictamente vinculados a la radiación solar y el tipo de suelo, respectivamente.

Los valores de altitud en la línea de bosque encontrados en este trabajo están estrechamente correspondidos con aquellos presentes en regiones intertropicales del planeta; es decir, entre el rango de 4000$4200 \mathrm{msnm}$. Las diferencias entre el límite de bosque continuo y el discontinuo, las cuales van desde 22 a 75 metros en promedio, se encuentran dentro del valor medio de la región intertropical del hemisferio norte como lo menciona Autio (2006); por lo que al menos para las regiones boreales intertropicales, los parámetros hallados en este trabajo podrían representar la referencia más actualizada.

La diferencia en la altitud de la línea de bosque cercana a 200 m entre los volcanes Iztaccíhuatl y Popocatépetl, a pesar de la cercanía entre ellos (14 km), debe ser una consecuencia de los distintos estados de actividad volcánica que poseen. Mientras que el Iztaccíhuatl está considerado en etapa de reposo (Macías et al., 2012) o incluso ha sido señalado como extinto (Mooser, 1958), el Popocatépetl ha permanecido activo recientemente, en un estado de constantes episodios eruptivos desde el año 1994 (Martin-Del Pozzo, 2012). Los flujos piroclásticos y de lava, principalmente, aunado con el alto calor geotérmico pueden ser los causantes del impedimento en la elevación de la línea de bosque en este edificio volcánico. Situaciones semejantes ocurren con el Nevado de Colima, perteneciente al Complejo Volcánico Colima que es considerado actualmente como el complejo más activo del país (Norini et al., 2010). Las situaciones relativamente similares de actividad volcánica entre estas dos montañas condicionan que la altitud máxima para el bosque continuo y discontinuo sea muy semejante entre ellos. 
Es probable que la mayor altitud del límite superior de bosque alcanzada en las vertientes orientales de todas las montañas estudiadas se deba en buena medida a las corrientes de los vientos alisios que traen consigo humedad proveniente del Golfo de México (Jáuregui, 1975). Esta humedad contenida en el aire algunas veces es depositada directamente en la vegetación y sobre el suelo, favoreciendo su hidratación. Adicionalmente, los vientos alisios y su humedad contenida sufren un forzamiento ascendente en las laderas del este que propicia su condensación, dando pie a la precipitación de tipo orográfico, beneficiando una vez más la hidratación vegetal. En las vertientes occidentales por su parte, donde se registra la altitud más baja de la línea de bosque de las cuatro vertientes, puede ser una consecuencia de la alta tasa de radiación solar justo después del mediodía, ocasionando el estrés fisiológico de la vegetación en combinación con las bajas temperaturas, tal como lo manifiestan Bader et al., (2007) y Bader y Ruijten (2008). Adicionalmente, debido a que la precipitación de tipo orográfico ocurre mayormente en las vertientes orientales, donde inciden los alisios, al cruzar la barrera orográfica estos vientos descienden por las laderas occidentales mayormente secos, propiciando el contraste de humedad entre ambas vertientes y la consecuente diferencia de hidratación vegetal.

Desde la perspectiva climática, el patrón de distribución de altitud máxima del límite de bosque parece obedecer condiciones de temperatura y precipitación relativamente semejantes entre las montañas analizadas. Sin embargo, el régimen térmico muestra valores un tanto menos homogéneos en comparación con la variable pluvial. Mientras que el modelo de distribución de la precipitación en regiones mexicanas de alta montaña, elaborado por Soto-Molina et al., (2020), indica una disminución cuasi lineal en función de la altura por arriba de 3000 msnm, con una precipitación anual acumulada inferior a $1000 \mathrm{~mm}$ entre $4000 \mathrm{y}$ $4100 \mathrm{msnm}$, la variable térmica por su parte, muestra un comportamiento distinto. De acuerdo con datos de temperatura media del aire en el volcán Citlaltépetl, reportados por Soto-Molina y Delgado-Granados (2020), el límite medio de individuos aislados (bosque discontinuo) coincide con un valor de $6.7^{\circ} \mathrm{C}$ y registra un número de 101 ciclos diurnos de helada al año. Por su parte, a partir de los registros de la estación San Juan Tetela (3393 msnm) y del gradiente altitudinal de temperatura determinado por Soto y Delgado (2020), en el volcán Iztaccíhuatl el mismo límite muestra una temperatura de $6.2^{\circ} \mathrm{C}$; a esta cota Andrés et al., (2010) reportan 56 ciclos de helada a nivel del suelo. Final- mente en el volcán Xinantécatl, a partir de la estación Nevado de Toluca (4283 msnm) y del gradiente térmico estimado (Soto y Delgado, 2020), el límite máximo altitudinal del bosque se posiciona sobre la isoterma $5.1^{\circ} \mathrm{C}$; los registros de la estación climatológica señalan 170 ciclos de helada al año.

Con base en lo anterior se enfatiza que el régimen térmico afecta la fisiología de la vegetación e impide su crecimiento, como lo señalan Wieser y Tausz (2007) y Hatfield y Prueger (2015). Los valores de temperatura, si bien son un tanto distintos entre las montañas mencionadas, justificando en parte su posición dentro de los factores que determinan la altitud del bosque, se aproximan al correspondiente de $5^{\circ} \mathrm{C}$ señalado por Lauer y Klaus (1975) como frontera superior del bosque en las montañas tropicales. No obstante lo anterior, al combinarse la temperatura con la variable de precipitación, identificada ésta como el componente más determinante, y aunado también a la tasa de insolación, se robustece la hipótesis de que el clima, como conjunto de variables, es el que rige principalmente la altitud del límite de bosque en las montañas mexicanas.

\section{CONCLUSIONES}

Los límites de bosque continuo y discontinuo en las montañas mexicanas son producto, en orden de importancia, de la precipitación, el tipo de suelo y de la incidencia de la radiación solar; en menor medida influye el basamento rocoso y la temperatura del aire. La altitud del límite de bosque en México se ubica dentro del rango correspondiente para las altas montañas intertropicales. La humedad del Golfo de México, transportada por los vientos alisios, determina que en las laderas orientales alcancen la cota más alta.

Se requiere de estudios futuros a nivel de micro escala para identificar con mayor precisión los factores que rigen la altitud del límite continuo y discontinuo del bosque en México. No obstante lo anterior, los resultados encontrados en este trabajo serán un buen referente desde la perspectiva forestal y climática para estudios posteriores donde se aborden los impactos del cambio climático en ecosistemas de alta montaña.

\section{AGRADECIMIENTOS}

El primer autor agradece al Consejo Nacional de Ciencia y Tecnología (CONACyT) por el apoyo financiero durante la estancia posdoctoral que facilitó este trabajo. 
A la Maestría en Ciencias del Ambiente de la Universidad Veracruzana, campus Poza Rica-Tuxpan por la estancia posdoctoral.

\section{BIBLIOGRAFÍA}

Aas, B. and Faarlund, T. (1996). The present and the Holocene birch belt in Norway. Paläo-klimaforschung (20), 19-42.

Aguilera, N., T. M. Dow and R. Hernández S. (1962). Suelos, problema básico en silvicultura. En: FAO (Ed.) Seminario y viaje de estudio de coníferas latinoamericanas. (pp. 108-140). México, D. F., México: Inst. Nac. Invest. Forest.

Alfaro-Ramírez, F. U., Arredondo-Moreno, J. T., PérezSuárez, M., and Endara-Agramont, Á. R. (2017). Pinus hartwegii Lindl. treeline ecotone: structure and altitudinal limits at Nevado de Toluca, Mexico. Revista Chapingo. Serie Ciencias Forestales y del Ambiente, 23(2), 261-273. https://doi. org/10.5154/r.rchscfa.2016.10.055

Andrés, N., Estremera, D. P., Zamorano, J. J., y VázquezSelem, L. (2010). Distribución del permafrost e intensidad de los procesos periglaciares en el estratovolcán Iztaccíhuatl (México) 1. Eria, (83), 291-310.

Arekhi, M., Goksel, C., Balik Sanli, F., and Senel, G. (2019). Comparative Evaluation of the Spectral and Spatial Consistency of Sentinel-2 and Landsat-8 OLI Data for Igneada Longos Forest. ISPRS International Journal of Geo-Information, 8(2), 56. https://doi.org/10.3390/ijgi8020056

Arno, S. F. (1984). Timberline, mountain and arctic frontiers. Seattle, WA., USA: The Mountaineers, $310 \mathrm{pp}$.

Arya, P. S. (2001). Introduction to micrometeorology (Vol. 79). Seattle, WA., USA: Academic Press, 420 pp.

Autio, J. (2006). Environmental factors controlling the position of the actual timberline and treeline on the fells of Finnish Lapland. Oulu, Finland: OULU University Press, 63 pp.

Bader, M. Y. and Ruijten, J. J. A. (2008). A topography-based model of forest cover at the alpine treeline in the tropical Andes. J. Biogeogr. (35), 711-723. https://doi.org/10.1111/j.13652699.2007.01818.x

Bader, M. Y., van Geloof, I. and Rietkerk, M. (2007).
High solar radiation hinders tree regeneration above the alpine treeline in northern Ecuador. Plant Ecol. (191), 33-45. https://doi.org/10.1007/ s11258-006-9212-6

Bharti, R. R., Rai, I. D., Adhikari, B. S., and Rawat, G. S. (2011). Timberline change detection using topographic map and satellite imagery: a critique. Tropical Ecology, 52(1), 133-137.

Braunisch, V., Patthey, P., and Arlettaz, R. (2016). Where to combat shrub encroachment in alpine timberline ecosystems: combining remotely sensed vegetation information with species habitat modelling. PloS one, 11(10), e0164318. https://doi.org/10.1371/journal.pone.0164318

Cairns, D. M. (1998). Modeling controls on pattern at alpine treeline. Geogr. Environ. Model. 2 (1), 43-63.

Chen, B. X., Sun, Y. F., Zhang, H. B., Han, Z. H., Wang, J. S., Li, Y. K., and Yang, X. L. (2018). Temperature change along elevation and its effect on the alpine timberline tree growth in the southeast of the Tibetan Plateau. Advances in Climate Change Research, 9(3), 185-191. https://doi.org/10.1016/j. accre.2018.05.001

Comisión Nacional para el Conocimiento y Uso de la Biodiversidad, CONABIO (8 de agosto de 2019). Portal de geoinformación. Sistema nacional de información sobre biodiversidad. Recuperado de http://www.conabio.gob.mx/informacion/ gis/?vns=gis_root/topog/infgrt/indi50kgw

Dahms, A. (1992). Wachstumsbedingungen bei Picea engelmannii (Parry) Engelm. und Abies lasiocarpa (Hook) Nutt. an unterschiedlich windexponierten Standorten im Waldgrenzbereich der Colorado Front Range, U.S.A. Diss. Mathematisch-Naturwissenschaftliche Fakultät, Westfälische Wilhelms-Universität, Münster.

Danzeglocke, J. (2005). Remote sensing of upper timberline elevation in the Alps on different scales. In proc. 24th EARSeL Symp. New Strategies for European Remote Sensing. 25-27th May 2004.

Delegido, J., Tenjo, C., Ruiz, A., Pereira, M., Pasqualotto, N., Gibaja, G. and Sanchis, J. (2016). Aplicaciones de Sentinel-2 a estudios de vegetación y calidad de aguas continentales. In Conference: XVII Simposio Internacional En Percepción Remota Y Sistemas de Información Geográfica (SEL$P E R)$. Puerto Iguazú, Argentina. 
Demant, A. (1978). Características del Eje Neovolcánico Transmexicano y sus problemas de interpretación. Revista mexicana de ciencias geológicas, 2(2), 172-187.

European Space Agency, ESA (2015). Sentinel-2 User Handbook. Issue 1 Rev. 2. 64 pp.

Ferrari, L., Orozco-Esquivel, T., Manea, V., and Manea, M. (2012). The dynamic history of the TransMexican Volcanic Belt and the Mexico subduction zone. Tectonophysics, 522, 122-149. https://doi. org/10.1016/j.tecto.2011.09.018

French, H. M. (2018). The periglacial environment (4th edition). Chichester, UK: John Wiley \& Sons. 513 pp.

Gaire, N. P., Koirala, M., Bhuju, D. R., and Carrer, M. (2017). Site-and species-specific treeline responses to climatic variability in eastern Nepal Himalaya. Dendrochronologia, 41, 44-56. https://doi. org/10.1016/j.dendro.2016.03.001

García, E. (2004). "Modificaciones al sistema de clasificación climática de Köppen (Para adaptarlo a las condiciones de la República Mexicana)" 5ạ edición. Instituto de Geografía, Universidad Nacional Autónoma de México, México. (No. C/551.6972 G3/2004), 90 pp.

Gellhorn, J. (2002). Song of the alpine: The Rocky Mountain tundra through the seasons. Boulder, Co. USA: Big Earth Publishing. 263 pp.

Gernandt, D. S., and Pérez-de la Rosa, J. A. (2014). Biodiversidad de Pinophyta (coníferas) en México. Revista mexicana de biodiversidad, 85, 126-133. https://doi.org/10.7550/rmb.32195

Hatfield, J. L., and Prueger, J. H. (2015). Temperature extremes: Effect on plant growth and development. Weather and climate extremes, 10, 4-10. https://doi.org/10.1016/j.wace.2015.08.001

Hoch, G. and Körner, C. (2003). The carbon charging of pines at the climatic treeline: A global comparison. Oecologia, (135), 10-21. https://doi. org/10.1007/s00442-002-1154-7

Hofgaard, A. (1997). Structural changes in the foresttundra ecotone: a dynamic process. In Past and future rapid environmental changes (pp. 255263). Berlin, Heidelberg: Springer. https://doi. org/10.1007/978-3-642-60599-4_20

Holtmeier, F. K. (2009). Mountain timberlines: ecology, patchiness, and dynamics (Vol. 36). Geneva,
Switzerland: Springer Science \& Business Media. $445 \mathrm{pp}$

Hreško, J., Bugár, G., and Petrovič, F. (2009). Changes of vegetation and soil cover in alpine zone due to anthropogenic and geomorphological processes. Landform Analysis, 10, 39-43.

Instituto Nacional de Estadística, Geografía e Informática, INEGI (2017). "Anuario estadístico y geográfico de los Estados Unidos Mexicanos". Aguascalientes, Ags. Méx: INEGI. 1066 pp.

Instituto Nacional de Estadística, Geografía e Informática, INEGI (7 de agosto de 2019). Continuo de Elevaciones Mexicano. Recuperado de https://www. inegi.org.mx/app/geo2/elevacionesmex/

Jandl, N., Jandl, R., and Schindlbacher, A. (2018). Future management options for cembran pine forests close to the alpine timberline. Annals of Forest Science, 75(3), 81. https://doi.org/10.1007/ s13595-018-0760-4

Jáuregui, O., E. (1975). Los sistemas de tiempo en el Golfo de México y su vecindad. Investigaciones geográficas, (6), 7-36.

Kaczka, R. J., Lempa, M., Czajka, B., Janecka, K., Rączkowska, Z., Hreško, J., and Bugar, G. (2015). The recent timberline changes in the Tatra Mountains: A case study of the Mengusovská Valley (Slovakia) and the Rybi Potok Valley (Poland). Geographia Polonica, 88(2), 71-83. http:// dx.doi.org/10.7163/GPol.0016

Kogan, F., Gitelson, A., Zakarin, E., Spivak, L., and Lebed, L. (2003). AVHRR-based spectral vegetation index for quantitative assessment of vegetation state and productivity. Photogrammetric Engineering and Remote Sensing, 69(8), 899-906.

Kullman, L. (1987). A decade of tree-line monitoring in the southern Swedish Scandes. UNGI Rep. (65), 91-202.

Lauer, W. and Klaus, D. (1975). Geoecological investigations on the timberline of Pico de Orizaba, Mexico. Arct. Alp. Res. 7 (4), 315-330.

Li, B. S. (1993). The alpine timberline of Tibet. In: (Alden, J., Mastrantonio, J. L. and Odum, S., Eds.) Forest development in cold climates: (pp. 511-527), New York, USA: Spinger. https://doi. org/10.1007/978-1-4899-1600-6_34

Li, Y., Chen, J., Ma, Q., Zhang, H. K., and Liu, J. (2018). Evaluation of Sentinel-2A surface reflectance de- 
rived using Sen2Cor in North America. IEEE Journal of Selected Topics in Applied Earth Observations and Remote Sensing, 11(6), 1997-2021. https://doi.org/10.1109/JSTARS.2018.2835823

Macías, J., Arce, J., García-Tenorio, F., Layer, P., Rueda, H., Reyes-Agustín, G., López-Pizaña, F., and Avellán, D. (2012). Geology and geochronology of Tlaloc, Telapón, Iztaccíhuatl, and Popocatépetl volcanoes, Sierra Nevada, central Mexico. In Aranda-Gómez, J.J., Tolson, G., and Molina-Garza, R.S., (Eds.) The Southern Cordillera and Beyond: Field Guide. 25. (pp. 163-193). Boulder, Co., USA: The Geological Society of America. https://doi. org/10.1130/2012.0025(08)

Malini, A. S., and Somashekar, R. K. (2013). Multispectral Monitoring of Vegetation Cover of Bangalore Metropolitan Area. Global Journal of Bio-Science and Biotechnology, 2(1), 27-32.

Malyshev, L. (1993). Levels of upper forest boundary in northern Asia. Vegetation (109), 175-186. https://doi.org/10.1007/BF00044749

Martin-Del Pozzo, A. L. (2012). Precursors to eruptions of Popocatépetl volcano, Mexico. Geofísica internacional, 51(1), 87-107.

Montero-García, I. A. (2004). Atlas arqueológico de la alta Montaña mexicana. México, D.F. Méx: SEMARNAT. $180 \mathrm{pp}$.

Mooser, F. (1958). Active volcanoes of Mexico. In Meyer, A. H. and MacBirney, A. R. (Eds). Catalogue of active volcanoes of the world including Solfatara Fields. Part VI. Mexico and Central America. Naples, Italy: International Volcanological Association. $36 \mathrm{pp}$.

Nelson, M. D., McRoberts, R. E., Holden, G. R., and Bauer, M. E. (2009). Effects of satellite image spatial aggregation and resolution on estimates of forest land area. International Journal of Remote Sensing, 30(8), 1913-1940. https://doi. org/10.1080/01431160802545631

Norini, G., Capra, L., Groppelli, G., Agliardi, F., Pola, A., and Cortes, A. (2010). Structural architecture of the Colima volcanic complex. Journal of Geophysical Research: Solid Earth, 115(B12). https://doi. org/10.1029/2010JB007649

Odland, A. (2015). Effect of latitude and mountain height on the timberline (Betula pubescens ssp. czerpanovii) elevation along the central Scandinavian mountain range. Fennia-International
Journal of Geography, 193(2), 260-270. https:// fennia.journal.fi/article/view/48291

Organización de las Naciones Unidas para la Agricultura y la Alimentación, ONUAA. (2010). Evaluación de los recursos forestales mundiales 2010, Informe Nacional, México. Roma, Italia: FAO. 98 pp.

Peres-Neto, P. R., Jackson, D. A., and Somers, K. M. (2005). How many principal components? Stopping rules for determining the number of nontrivial axes revisited. Computational Statistics \& Data Analysis, 49(4), 974-997. https://doi. org/10.1016/j.csda.2004.06.015

Reddy, A. S., and Reddy, M. J. (2013). NDVI based assessment of land use land cover dynamics in a rainfed watershed using remote sensing and GIS. International Journal of Scientific \& Engineering Research, 4(12), 87-93.

Rzedowski, J. (2006). Vegetación de México. Comisión Nacional para el Conocimiento y Uso de la Biodiversidad. México, D.F., Méx: Limusa. 504 pp.

Sader, S. A., and Winne, J. C. (1992). RGB-NDVI colour composites for visualizing forest change dynamics. International journal of remote sensing, 13(16), 3055-3067. https://doi. org/10.1080/01431169208904102

Salomons, J. B. (1986). Paloecology of volcanic soils in the Columbian central cordillera (Parque Nacional de los Nevados). Dissertationes Botanicae (95), 1-212.

Sánchez-González, A. (2008). Una visión actual de la diversidad y distribución de los pinos de México. Madera y bosques, 14(1), 107-120. https:// doi.org/10.21829/myb.2008.1411222

Santaella, W. (2013). Modelos espaciales de la dinámica de la vegetación en escenarios de cambio climático en el "Timberline» selva nubladaparamo de la vertiente norte de la Sierra Nevada de Mérida (Venezuela); escenario B1 y situación constante. Revista de Teledetección, 39, 63-82.

Schwörer, C., Henne, P. D., and Tinner, W. (2014). A model-data comparison of Holocene timberline changes in the Swiss Alps reveals past and future drivers of mountain forest dynamics. Global change biology, 20(5), 1512-1526. https://doi. org/10.1111/gcb.12456

Singh, C.P., Panigrahy, S and Thaplyal, A. (2012). Monitoring alpine treeline shift in parts of the Indian Himalayans using remote sensing. Current Sci- 
ence 102(4), 559-562. https://www.jstor.org/stable/24084105

Sitko, I., and Troll, M. (2008). Timberline changes in relation to summer farming in the Western Chornohora (Ukrainian Carpathians). Mountain Research and Development, 28(3), 263-272. https://doi. org/10.1659/mrd.0963

Soto-Molina, V., Yoshikawa, K., and Schörghofer, N. (2020). Climatic variation in the high-mountain of central Mexico: temperature and precipitation indices at the Nevado de Toluca volcano. Atmósfera. https://doi.org/10.20937/ATM.52768

Soto-Molina, V. H. y Delgado-Granados, H. (2020). Dos años de observaciones de temperatura superficial del aire y del suelo en la vertiente norte del volcán Pico de Orizaba, México. Acta Universitaria, 30(1), 1-16. https://doi.org/10.15174/au.2020.2427

Soto, V. y Delgado, H. (2020). Estimación de la temperatura del aire en la alta montaña mexicana mediante un modelo de elevación del terreno: caso del volcán Nevado de Toluca (México). Ería. Revisa Cuatrimestral de Geografía 2020(2), 167-182. https://doi.org/10.17811/er.2.2020.167-182

Steinmann, V. W., Amezcua, L. A., Amezcua, Y. R., Méndez, S. M. V., Cárdenas, R. A. H., y Yanes, Y. A. M. (2019). La vegetación alpina mexicana: islas frías sobre las nubes. Comisión Nacional para el Conocimiento de la Biodiversidad. México, D.F., Méx: CONABIO. 20 pp.

Takahashi, K., Hirosawa, T., and Morishima, R. (2012). How the timberline formed: altitudinal changes in stand structure and dynamics around the timberline in central Japan. Annals of botany, 109(6), 1165-1174. https://doi.org/10.1093/aob/mcs043
Turunen, M., and Latola, K. (2005). UV-B radiation and acclimation in timberline plants. Environmental Pollution, 137(3), 390-403. https://doi. org/10.1016/j.envpol.2005.01.030

United States Geological Survey, USGS (5 de agosto de 2019). EarthExplorer. Recuperado de https:// earthexplorer.usgs.gov/

Vincze, I., Orbán, I., Marinova, E., Jakab, G., Birks, H. H., Finsinger, W., and Magyari, E. K. (2014). Drivers of Holocene treeline and timberline changes in the Retezat Mountains (South Carpathians, Romania). GEOREVIEW: Scientific Annals of Stefan cel Mare University of Suceava. Geography Series, 24(2), 184-185.

Wang, T., Zhang, Q. B., and Ma, K. (2006). Treeline dynamics in relation to climatic variability in the central Tianshan Mountains, northwestern China. Global Ecology and Biogeography, 15(4), 406-415. https://doi.org/10.1111/j.1466822X.2006.00233.x

Ward, J. V. (1994). Ecology of alpine streams. Freshwater biology, 32(2), 277-294. https://doi. org/10.1111/j.1365-2427.1994.tb01126.x

Wieser, G. and Tausz, M. (Eds). (2007). Trees at their upper limit. Treelife limitation at the alpine timberline. Dordrecht, the Netherlands: Springer. 232 pp.

Zaitunah, A., Ahmad, A. G., and Safitri, R. A. (2018). Normalized difference vegetation index (ndvi) analysis for land cover types using landsat 8 oli in besitang watershed, Indonesia. In IOP Conference Series: Earth and Environmental Science. 126 (1), 012112. https://iopscience.iop.org/article/10.1088/1755-1315/126/1/012112 